\title{
Tumor adenomatoide de epidídimo: El tumor más frecuente de las estructuras paratesticulares
}

\author{
Bestard Vallejo JE, Tremps Velázquez E, Blázquez Mañá C*, Celma Doménech A, \\ de Torres Ramírez I*, Morote Robles J.
}

Servicio de Urología y *Anatomía Patológica del Hospital General Universitario Vall d'Hebron. UAB. Barcelona.

Actas Urol Esp. 2008;32(6):611-617

\section{RESUMEN}

TUMOR ADENOMATOIDE DE EPIDÍDIMO: EL TUMOR MÁS FRECUENTE DE LAS ESTRUCTURAS PARATESTICULARES

Introducción: Los tumores paratesticulares son neoplasias poco frecuentes aunque habitualmente benignas que suelen ser tributarias de extirpación local. Dentro de ellos se considera que los tumores adenomatoides de epidídimo constituyen el subgrupo más frecuente.

Objetivos: Analizar los casos de tumores paratesticulares intervenidos en nuestro centro, así como el diagnóstico y tratamiento de los tumores adenomatoides de epidídimo.

Material y métodos: Se revisan de forma retrospectiva 8 pacientes con tumores paratesticulares intervenidos entre julio de 1997 y julio 2007. Se analiza en cada caso la forma de presentación, la orientación diagnóstica que aportaba la prueba de imagen, el tratamiento indicado para cada paciente y el diagnóstico definitivo.

Resultados: La edad media de los pacientes fue de 44,1 años (22-69), consultando la mayoría de ellos por tumoración escrotal de aparición subaguda que presentaban en la ecografía un tamaño medio de 2,8 cm (1,5-7). Se practicó la extirpación local en todos los casos a excepción de un paciente con sospecha de un teste accesorio malignizado y otro con una lesión aparentemente intratesticular de gran tamaño. Sólo en dos casos se practicó una biopsia peroperatoria. El diagnóstico fue en 4 casos de tumor adenomatoide de epidídimo, en dos casos de pseudotumor fibroso de epidídimo, en un caso de leiomioma de epidídimo y en otro de angiolipoma de cordón espermático. Sólo en un caso diagnosticado de tumor adenomatoide de epidídimo la ecografía informaba de tumoración sólida sugestiva del diagnóstico definitivo.

Conclusiones: Los tumores adenomatoides de epidídimo son tumores poco frecuentes que se pueden diagnosticar a cualquier edad. La ecografía puede orientar el diagnóstico pero su capacidad para diferenciarlos es baja. La naturaleza habitualmente benigna tanto del tumor adenomatoide de epidídimo como del resto de tumores paratesticulares obligaría a practicar como primera opción la extirpación local de los mismos y, ante cualquier duda, debería practicarse una biopsia peroperatoria.

Palabras clave: Epidídimo. Tumores paratesticulares. Tumor adenomatoide de epidídimo.

\section{ABSTRACT}

\section{ADENOMATOID TUMOUR OF EPIDIDYMIS: THE MOST COMMON TUMOUR OF THE PARATESTICULAR} STRUCTURES

Introduction: Paratesticular tumours are rare but generally benign neoplasms, usually treated by local excission. Adenomatoid tumours of epididymis are the most common of these tumours.

Objectives: Analyze paratesticular tumours treated in our center and describe dyagnosis and treatment of adenomatoid tumours of epididymis.

Material and Methods: We retrospectively review 8 patients with paratesticular tumours treated from July 1997 to July 2007. We analyze clinical presentation, dyagnostic suspicion given by image technique, treatment followed and final dyagnosis.

Results: Patients median age was 44.1 years (22-69), presenting most of them subacute scrotal tumour with median size by ultrasound of $2.8 \mathrm{~cm}(1.5-7)$. All of them were locally extirpated except one with suspicion of a malignant polyorchidism and another one with an apparently intratesticular lesion of great size. Just in two cases peroperatory biopsy was performed. Dyagnosis was in 4 cases adenomatoid tumour of epididymis, in two cases fibrous pseudotumour of epididymis, in one case leiomyoma of epididymis and in one case angiolipoma of spermatic cord. Just in one case diagnosed of adenomatoid tumour of epididymis ultrasound confirmed solid tumour suggesting the final dyagnosis.

Conclusions: Adenomatoid tumors of epididymis are rare tumours which may present at any age. Ultrasound may help in dyagnosis, but its capacity to distinguish this lesions is low. Benignity of adenomatoid tumour of epididymis as well as most of the other paratesticular tumours should make local excission the treatment of choice and, when any doubt existed, peroperatory biopsy should be performed. 
$\mathrm{L}_{\mathrm{p}}^{\mathrm{o}}$ os tumores paratesticulares (TP) son entidades poco frecuentes aunque habitualmente benignas que, si se diagnostican correctamente, son tributarias de extirpación local. Dentro de ellos se considera que los tumores adenomatoides de epidídimo (TAE) constituyen el subgrupo más frecuente ${ }^{1}$.

Hemos llevado a cabo la revisión de los TP tratados en nuestro centro durante los últimos diez años, revisando en cada caso si la orientación diagnóstica fue la correcta, cuántos de ellos resultaron TAE y el tratamiento indicado para cada uno. Se practica igualmente una revisión de la literatura sobre el diagnóstico y manejo de dichos tumores.

\section{MATERIAL Y MÉTODOS}

Se practica un estudio retrospectivo de los TP registrados en nuestros archivos informáticos entre el 1 de julio de 1997 y el 1 de julio 2007. Se analiza en cada caso la forma de presentación, la orientación diagnóstica que aportaba la prueba de imagen y el tratamiento indicado para cada paciente. Se describe igualmente el informe anatomopatológico y el seguimiento de cada caso.

\section{RESULTADOS}

Un total de 8 pacientes han sido intervenidos de TP en nuestro centro durante los últimos 10 años. La edad media de los pacientes fue de 44,1 años (rango: 22-69) y la mayoría de ellos consultaron por tumoración escrotal de aparición subaguda y más o menos dolorosa. Sólo un paciente de 28 años debutó como un cuadro de orquiepididimitis que requirió de varias consultas a urgencias por dolor escrotal agudo, sin fiebre ni síndrome miccional. En dicho paciente los urocultivos resultaron siempre negativos y la analgesia y el tratamiento antibiótico sólo lograron una mejoría relativa de la clínica.
En ningún caso los pacientes refirieron historia de traumatismos ni de infecciones previas del tracto urinario. La ecografía fue en todos los casos la única prueba de imagen que se solicitó (Fig. 1). El tamaño medio de las lesiones era de $2,8 \mathrm{~cm}$ (rango: 1,5-7). Las características clínicas y ecográficas de cada caso se describen en la Tabla 1.

Sólo a un paciente de 22 años se le solicitó niveles séricos de alfa fetoproteína, gonadotropina coriónica humana y lactato deshidrogenasa para descartar el origen germinal del tumor, los cuales resultaron negativos.

Cinco de los ocho casos $(62,5 \%)$ se intervinieron bajo el diagnóstico ecográfico de TP. En la Tabla 2 se describen las características anatomopatológicas de cada tumor y el abordaje quirúrgico utilizado en cada caso.

El TAE fue la neoplasia diagnosticada con mayor frecuencia (4 de 8,50\%). Se trataban de nódulos bien delimitados, blanquecino-grisáceos y de consistencia elástica-homogénea al corte, sin áreas de necrosis ni hemorragias (Fig. 2). Microscópicamente

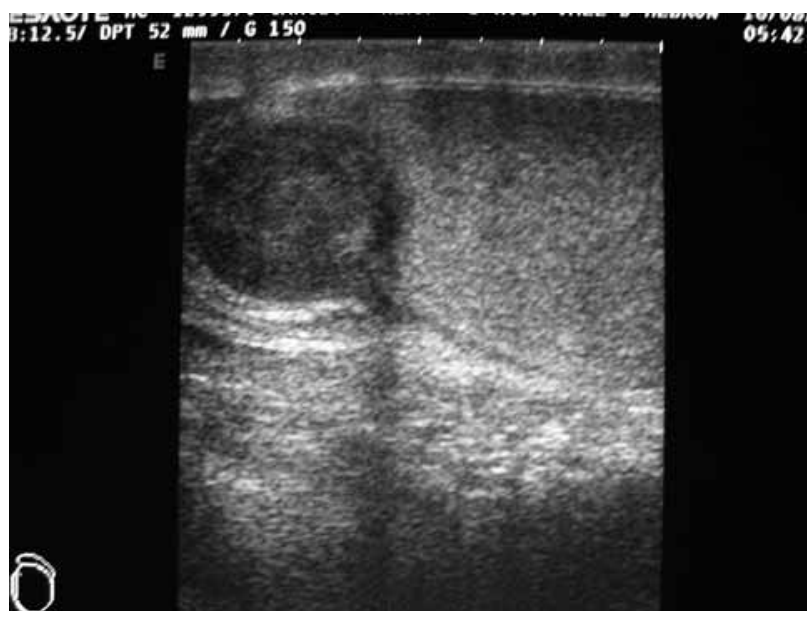

FIGURA 1. Imagen hiperecogénica de $1,5 \mathrm{~cm}$ en polo superior de epididimo en paciente con TAE.

Tabla 1. Presentación y ecografía

\begin{tabular}{|c|c|c|c|c|c|c|}
\hline Caso & Edad (años) & Lateralidad & Ecografia & Zona & Tamaño $(\mathrm{cm})$ & Hidrocele \\
\hline 1 & 26 & Derecho & Poliorquitis & Epidídimo & 2 & No \\
\hline 2 & 47 & Derecho & Tumor sólido & Epidídimo & 1,6 & No \\
\hline 3 & 67 & Izquierdo & Formación quística & Teste & 7 & $\mathrm{Si}$ \\
\hline 4 & 28 & Derecho & Epididimitis crónica & Epidídimo & 1,5 & No \\
\hline 5 & 22 & Derecho & Tumor sólido & Epidídimo & 2 & No \\
\hline 6 & 43 & Izquierdo & Tumor sólido & Epidídimo & 2,6 & No \\
\hline 7 & 51 & Derecho & Tumor sólido & Epidídimo & 3 & No \\
\hline 8 & 69 & Izquierdo & Lipoma & Cordón & 2 & No \\
\hline
\end{tabular}


Tabla 2. Diagnóstico y tratamiento

\begin{tabular}{|c|c|c|c|c|c|c|}
\hline Caso & Cirugia & Via & Peroperatoria & Diagnóstico & IHQ & Invasión \\
\hline 1 & Orquiectomía & Inguinal & No & TAE & No & No \\
\hline 2 & Tumorectomía & Transescrotal & No & TAE & $\mathrm{Si}$ & No \\
\hline 3 & Orquiectomía & Transescrotal & No & TAE & $\mathrm{Si}$ & No \\
\hline 4 & Tumorectomía & Transescrotal & $\mathrm{Si}$ & TAE & $\mathrm{Si}$ & $\mathrm{Si}$ \\
\hline 5 & Tumorectomía & Transescrotal & No & PFE & $\mathrm{Si}$ & No \\
\hline 6 & Tumorectomía & Transescrotal & $\mathrm{Si}$ & PFE & $\mathrm{Si}$ & No \\
\hline 7 & Tumorectomía & Transescrotal & No & Leiomioma & $\mathrm{Si}$ & No \\
\hline 8 & Tumorectomía & Inguinal & No & Angiolipoma & No & No \\
\hline
\end{tabular}

TAE: Tumor adenomatoide de epidídimo; PFE: Pseudotumor fibroso de epidídimo; IHQ: Inmunohistoquímica.

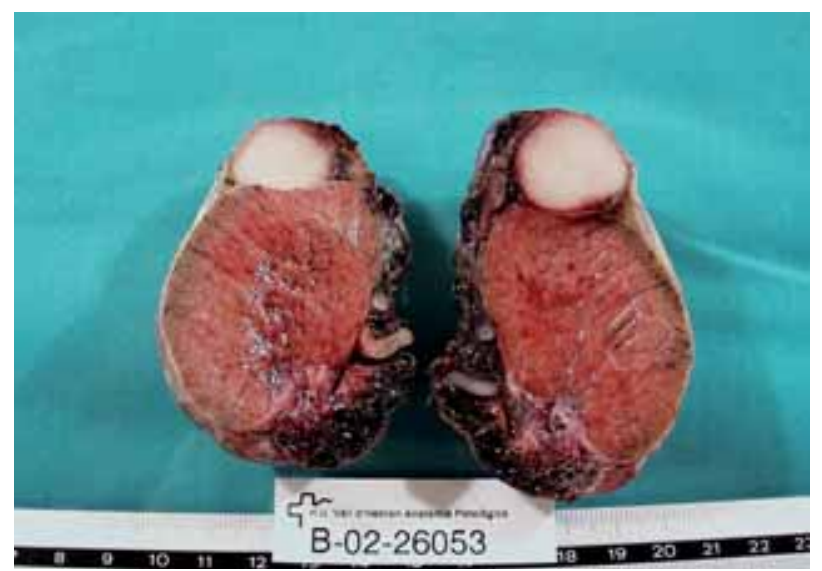

FIGURA 2. Sección macroscópica de pieza de orquiectomia mostrando en polo inferior una tumoración bien delimitada, blanquecina y elástica, de localización paratesticular. se describian tumoraciones bien delimitadas aunque no encapsuladas, constituidas por células de citoplasma discretamente acidófilo y marcadamente vacuolizado, sin nucleolo ni actividad mitótica. Dichas células se disponian en un patrón tubular entremezclándose con un estroma fibromuscular discretamente hialinizado y observándose entre ellas algún grupo de fibras musculares lisas (Fig. 3). Uno de los casos presentaba invasión del tejido testicular adyacente. Se solicitaron técnicas inmunohistoquímicas para el diagnóstico de tres de los TAE, observándose intensa positividad para la calretinina en el 100\% de las células tumorales (Fig. 4). En un caso se demostró positividad para actina y en otro para vimentina en las células estromales. CEA, CD 31, EMA y CD 34 fueron negativos en todas las células.

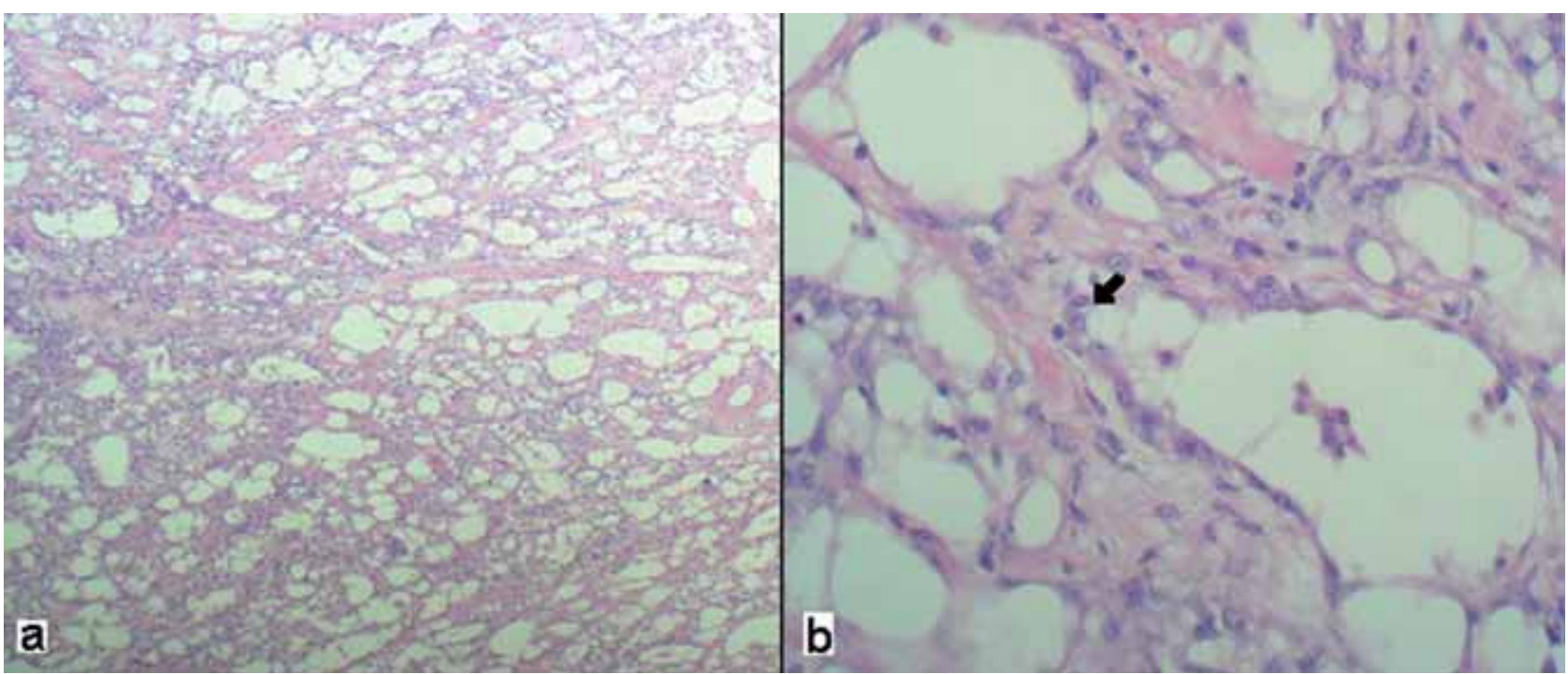

FIGURA 3. a) Histológicamente muestra la clásica morfología tubular y cordonal con estructuras irregulares y dilatadas, con células vacuoladas que se disponen entre un estroma hialinizado (X4OH\&E). b) Las formaciones tubulares están tapizadas por células monótonas (flecha) de citoplasma eosinófilo y núcleo grande con nucleolo prominente (X250H\&E). 


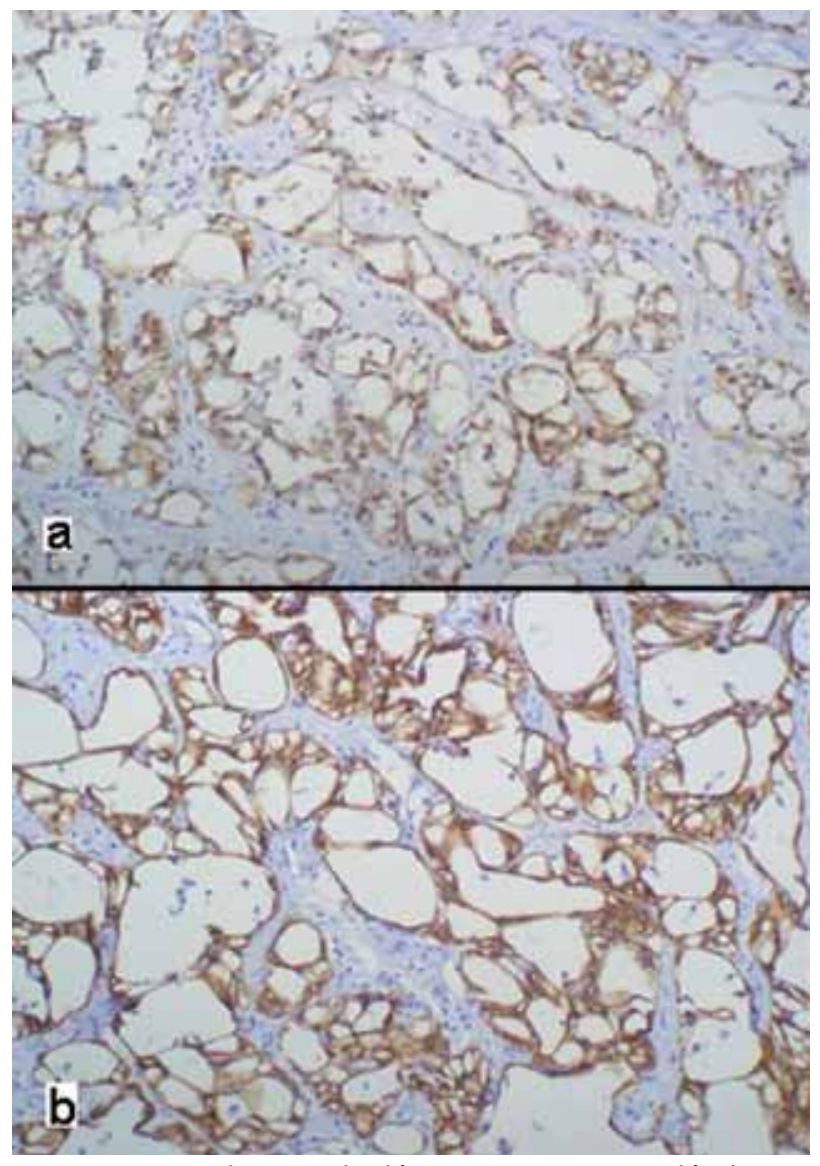

FIGURA 4. Con inmunotinción se observa expresión intensa para calretinina (a) y citoqueratinas (b) apoyando el origen mesotelial del tumor adenomatoide (X100).

Ninguno de los cuatro pacientes diagnosticados de TAE presentaron recidiva de la enfermedad durante el seguimiento. Un paciente transplantado pulmonar falleció a los 3 meses de la intervención por sobreinfección respiratoria, y otro paciente con ventrículo único falleció a los 50 meses por un episodio de infarto agudo de miocardio. El resto de pacientes diagnosticados de TP se encuentran igualmente libres de enfermedad en la actualidad. El tiempo medio de seguimiento fue de 45,5 meses (rango: 1-94).

\section{DISCUSIÓN}

Llamamos región paratesticular a aquella formada por el cordón espermático, las túnicas testiculares (vaginal externa, interna y albugínea), el epidídimo y las estructuras anejas de testes y epidídimos ${ }^{2}$.

El TAE comprende el 30\% de los tumores de estas estructuras y constituye el 60-70\% del total de neoplasias benignas que se desarrollan en las mismas. Se ha postulado que la inflamación puede tener algún papel en el desarrollo de estos tumores debido a su asociación en ocasiones a periorquitis e hidrocele y también por la presencia en su seno de células inflamatorias ${ }^{2}$.

El primer tumor benigno de epidídimo fue descrito por Sakaguchi en $1916^{3}$, siendo Golden y Ash ${ }^{4}$ en 1945 los que por primera vez acuñaron la denominación de TAE.

Beccia et al. ${ }^{1}$ recogieron en 1976 un total de 314 tumores de epidídimo, de los cuales el $75 \%$ eran benignos y, de ellos, el 73\% resultaron TAE (el 55\% del total), seguidos de los leiomiomas (11\%) y de los cistoadenomas papilares (9\%). El 7\% restante lo componían otras entidades como angiomas, lipomas, fibromas, hamartomas, teratomas y colesteatomas.

En un 15\% de los casos los TAE aparecen de forma bilateral, siendo cuatro veces más frecuente su aparición en el polo inferior y presentando una ligera predominancia por el epidídimo izquierdo ${ }^{5}$. Si bien la mayoría suelen diagnosticarse entre la tercera y quinta década de la vida, la literatura española describe un caso en un paciente de 70 años $^{6}$.

La localización más frecuente de estos tumores es el tracto genital masculino (básicamente epidídimo, seguido de túnica vaginal y cordón espermático), y a pesar de que también pueden hallarse en el aparato genital femenino, la relación es de 5 a 3,5 a favor de los hombres ${ }^{7}$.

En las mujeres la localización más frecuente del tumor adenomatoide son el útero y las trompas de Falopio. La localización intrapélvica de estos tumores puede hacer que alcancen tamaños mucho mayores que los del sexo masculino de forma relativamente asintomática, como el tumor adenomatoide de $15 \mathrm{~cm}$ de diámetro publicado por Hanada et $a .^{8}$ y que afectaba útero, ovario y apéndice.

El porqué de esta mayor incidencia en el área genital se desconoce. Han sido descritos también tumores adenomatoides en órganos extragenitales como las glándulas adrenales, ganglios linfáticos, mediastino, corazón, páncreas, y pleura ${ }^{7}$.

En el estudio anatomopatológico se suele describir como una tumoración de entre 1 y $5 \mathrm{~cm}$, redondeada, firme, de consistencia elástica y bien delimitada.

Microscópicamente su apariencia no es la típica de las neoplasias benignas: se trata de un tumor no encapsulado y que puede infiltrar el testículo adyacente hasta en un $25 \%$ de los casos (hasta en un 
$40 \%$ cuando se trata de tumores del polo superior). Aún así, nunca se han descrito metástasis ni recidivas tras su excisión, lo que confirmaría su naturaleza benigna.

Las células que lo forman pueden ir de aplanadas a cuboidales, formando cordones sólidos de aspecto epitelial. Su citoplasma se encuentra con frecuencia vacuolizado, con apariencia de células endoteliales sin positividad para mucinas epiteliales. El estroma circundante es de tipo fibroso, en ocasiones hialinizado, con agregados linfoides, fibras elásticas y células musculares lisas en su interior ${ }^{9}$.

Mostofi et al. ${ }^{10}$ fueron los primeros en describir tres tipos de patrones microscópicos: sólido (el más habitual), glandular (con la presencia de luces revestidas de células cúbicas) y angiomatoide (con amplios espacios tapizados por células de apariencia endotelial). En los tres casos su comportamiento es siempre benigno.

En el análisis inmunohistoquímico sus células expresan marcadores epiteliales, y de membrana (citoqueratinas AE1/AE3 y EMA respectivamente). Suelen presentar también grandes vacuolas con ácido hialurónico, sustancia que puede encontrarse en células mesoteliales.

El estudio inmunohistoquímico también demuestra el origen mesotelial mediante la positividad de la tinción para celretinina, una proteína fijadora de calcio de $29 \mathrm{kDa}$ que pertenece a la familia de la proteína S 100 y se localiza tanto en el citoplasma como en el núcleo celulares. Dicha proteína se identificó por primera vez en neuronas y tiene una alta sensibilidad para detectar tejido mesotelial ${ }^{11}$.

Otros hallazgos inmunohistoquímicos que permiten demostrar el origen mesotelial de estas células tumorales consisten en la negatividad para la expresión de marcadores epiteliales y endoteliales, como el CEA o el antígeno relacionado con el factor VIII ${ }^{9}$, además de HBME-1, MOC31, BER-EP4, B72.3 y $\mathrm{CD} 15^{7}$.

Ultraestructuralmente se pueden apreciar la presencia de microvellosidades apicales, complejos de unión intercelulares, canales intercelulares y haces de filamentos intracitoplasmáticos, todo ello también característico de las células mesoteliales ${ }^{12}$.

La ecografía es la prueba de imagen de elección ante la sospecha de cualquier masa a nivel testicular o paratesticular, siendo capaz de diferencia entre un origen intratesticular (malignas en su gran mayoría) o extratesticular (la mayor parte benignas) de la masa. El TAE se caracteriza por aparecer como una masa sólida, isoecogénica y bien delimitada. Sin embargo, la presencia de una arquitectura altamente homogénea que origina la ausencia de interfases intratumorales y la presencia en algunas ocasiones de una banda de tejido muscular liso que lo delimita pueden hacer pasar la imagen por un quiste ${ }^{13}$.

En nuestra serie, de los 4 TAE sólo 1 fue orientado como TP en el informe ecográfico, mientras que el resto de tumores sí fueron orientados correctamente como TP.

La TC y la RNM pueden ayudar también en el diagnóstico diferencial de estas lesiones. Así, el contenido graso de los lipomas de epidídimo aparecen con una densidad muy específica en la TC, y en la RNM se caracterizan por presentar una hiperseñal en T1 que los confiere un aspecto brillante. Por otra parte la RNM permite diferenciar la poliorquitis o duplicación testicular, de características iguales al teste normal (señal de intensidad intermedia en T1 y aumentada en $\mathrm{T} 2$ ) y rodeada por una capa hipointensa tanto en $\mathrm{T} 1$ como en $\mathrm{T} 2$ que corresponde a la túnica albugínea ${ }^{5}$.

La única referencia en la literatura española en la que se practica una RNM a un TAE éste se describe como una masa de señal hipointensa en $\mathrm{T} 1$, mientras que en T2 aparece con un halo hipointenso y centro más hiperintenso ${ }^{14}$.

La localización intratesticular es la segunda localización mas frecuente de los tumores adenomatoides. Se originan a partir de las láminas vaginales o de la túnica albugínea y son también más frecuentes en el polo inferior ${ }^{15}$. Habitualmente la ecografía los identifica como un tumor periférico y bien delimitado. Dicha técnica permite diferenciar con relativa facilidad estos tumores de las neoplasias testiculares, especialmente los originados en la vaginal externa, gracias al líquido acumulado entre ésta y la vaginal interna. No distinguirá con tanta facilidad en cambio aquellos tumores que surjan de la vaginal interna o de la túnica albugínea ${ }^{16}$. La RNM puede identificar estas lesiones como masas que surgen de la superficie testicular, hipointensa respecto del parénquima circundante. También presentan menos captación de contraste que el testículo normal, en contra de lo que se esperaría de una tumoración maligna ${ }^{16}$. 
Muchos tratados defienden la exploración quirúrgica de las lesiones sólidas de epidídimo, sean o no sintomáticas, y rechazan las biopsias por punción de dichas masas por su facilidad de diseminación en el caso de malignidad ${ }^{17}$. Sin embargo, la frecuente benignidad de estas masas paratesticulares, así como el mal pronostico que tienen estas igualmente en los casos de malignidad, ha llevado a varios grupos a practicar sistemáticamente la punción con aguja fina de estas lesiones ${ }^{18}$.

En un estudio en el que se practico dicho procedimiento a 228 pacientes con nódulos de epidídimo el citólogo fue capaz de dar un diagnóstico en el 90\% de los casos, resultando uno solo de ellos una neoplasia maligna.

En los 4 casos diagnosticados de TAE el aspirado se caracterizaba por presentar células redondeadas muy homogéneas, colocadas en láminas y agregados poco cohesivos. Su núcleo era vesicular, finamente granular, con nucleolo pequeño y cromatina uniformemente distribuida, y presentaban algunas células fusiformes aisladas entre ellas ${ }^{19}$.

En nuestra serie se optó por la extirpación local de la lesión en la mayoría de los casos. Únicamente en un paciente de 26 años la sospecha de malignidad de un teste accesorio obligó a practicar una orquiectomía por vía inguinal, y en otro paciente de 67 años con una lesión intratesticular de gran tamaño se optó por una orquiectomía transescrotal. Ambos fueron diagnosticados finalmente de TAE. Sólo en dos casos se confirmó el diagnóstico mediante una biopsia peroperatoria.

A parte del TAE, otras posibles neoplasias benignas que tienen su origen en las estructuras paratesticulares son los leiomiomas, con sus células fusiformes típicas, y el cistoadenoma papilar, típico del síndrome de Von Hippel Lindau y en el que microscópicamente se identifican túbulos y quistes dilatados en los que se proyectan estructuras papilares $^{9}$. Los pseudotumores fibrosos son reacciones fibrosas proliferativas que nacen en su mayor parte de la túnica vaginal y crecen imitando una neoplasia. Macroscopicamente se observa una masa irregular compuesta por múltiples nódulos y microscópicamente esta formado por un tejido fibroso denso hialinizado, con acúmulo de tejido colágeno, fibroblastos y productos de inflamación crónica ${ }^{5}$.

Otras posibles lesiones que podemos encontrar a este nivel son los hemangiomas (que en los adultos resultan extremadamente difíciles de diferenciar del varicocele), el lipogranuloma escleroso (que suele aparecer tras la inyección de un cuerpo extraño en este nivel), el espermatocele (dilatación de un ductus eferente cerca de la cabeza del epidídimo), la epididimitis crónica (como la ocasionada por la tuberculosis) y los tumores neuroectodérmicos de la infancia $^{18}$.

Los tumores malignos primarios de epidídimo comprenden clásicamente el $25 \%$ de las tumoraciones de esta zona, si bien en medios donde existe una alta incidencia de tuberculosis epididimaria esta incidencia puede descender por debajo del $1 \% 19$. Pueden dividirse en tres grupos según sean sarcomas (fibrosarcoma, leiomiosarcoma y rabdomiosarcoma) tumores epiteliales (carcinomas epidermoides y glandulares) y tumores disembrionarios. Si el resultado histológico confirma la malignidad el tratamiento de elección es la orquiectomía inguinal, con linfadenectomía retroperitoneal según el análisis histológico y el resultado de la TC. Habitualmente el tratamiento debe completarse con radioterapia y quimioterapia, resultando un pobre pronóstico en la mayoría de los casos descritos ${ }^{17}$. Nuestra serie no describe ninguno de estos tumores en los últimos 10 años.

\section{CONCLUSIONES}

Los TAE son tumores poco frecuentes que se pueden diagnosticar en cualquier franja de edad. La ecografía puede orientar el diagnóstico pero en nuestra serie parece tener una baja capacidad para diferenciar dichas lesiones. La naturaleza habitualmente benigna tanto del TAE como del resto de TP obligaría a practicar la extirpación local de los mismos como tratamiento de elección, y ante la sospecha de una posible tumoración maligna, debería confirmarse el diagnóstico mediante una biopsia peroperatoria antes de practicarse la orquiectomía.

\section{REFERENCIAS}

1. Beccia DJ, Krane RJ, Olsson CA. Clinical management of nontesticular intrascrotal tumors. J Urol. 1976;116(4):476-479.

2. Tato Rodríguez J, Lema Grille J, Cimadevila García A, Abelenda Barca J, Blanco Parra M, Cimadevila Covelo A, et al. New report of epididymal adenomatoid tumor. Infrequent pathology. Actas Urol Esp. 1997.21(5):502-507.

3. Sakaguchi Y. Uber das Adenomyom des Nebenhodens. Ztschr Pathol. 1916;18(3):379-387.

4. Golden A. Ash J.E. Adenomatoid tumors of the genital tract. Am J Path, 1945.21(1):63-79. 
5. Akbar SA, Sayyed TA, Jafri SZ, Hasteh F, Neill JS. Multimodality imaging of paratesticular neoplasms and their rare mimics. Radiographics. 2003;23(6):1461-1476.

6. Morote J, Rabella A, Benasco C. Adenomatoid tumor of the epididymis. Arch Esp Urol. 1984;37(1):61-64.

7. Canedo-Patzi AM, León-Bojorge B, de Ortíz-Hidalgo C. Adenomatoid tumor of the genital tract. Clinical, pathological and immunohistochemical study in 9 cases. Gac Med Mex. 2006; 142(1):59-66.

8. Hanada S, Okumura Y, Kaida K, Multicentric adenomatoid tumors involving uterus, ovary, and appendix. J Obstet Gynaecol Res. 2003;29(4):234-238.

9. Kuhn MT, Maclennan GT. Benign neoplasms of the epididymis. J Urol. 2005;174(2):723.

10. Mostofi FK. Proceedings: Testicular tumors. Epidemiologic, etiologic, and pathologic features. Cancer. 1973;32(5):1186-1201.

11. Moyano Calvo JL, Giraldez Puig J, Sánchez de la Vega J, Dávalos Casanova G, Morales López A. Adenomatoid tumor of the epididymis. Actas Urol Esp. 2007;31(4):417-419.

12. Romero Pérez P, Amat Cecilia M, Rafie Mazketli W, Merenciano Cortina FJ, Laforga Canales JB. Epididymal adenomatoid tumor. Review of the national literature and report of a case. Actas Urol Esp. 1996;20(9):839-843.

13. Vick CW 3rd, Klein FA, Schneider V. Adenomatoid tumor of epididymis simulating benign cyst on scrotal ultrasound. Urology. 1991;38(4):369-371.
14. Serrano Frago P, Medrano Llorente P, Borque Fernando A, Pascual Llorente M, Allue López M. Consults by scrotum mass: epididymo lesions]. Actas Urol Esp. 2007;31(4):420-422.

15. Gökçe G, Kiliçarslan H, Ayan S, Yildiz E, Kaya K, Gültekin EY. Adenomatoid tumors of testis and epididymis: a report of two cases. Int Urol Nephrol. 2001;32(4):677-680.

16. Patel MD, Silva AC. MRI of an adenomatoid tumor of the tunica albuginea. AJR Am J Roentgenol. 2004;182(2):415-417.

17. Flores N, Infante R. Tumores paratesticulares. En Tratado de Urología (tomo IV) editores Jiménez Cruz JF y Rioja Sanz LA. (p. 2181-93)

18. Singh I, Dev G, Singh N. Chronic epididymitis (epididymal nodule) mimicking an adenomatoid tumor-case report with review of literature. Int Urol Nephrol. 2002;34(2):219-222.

19. Gupta N, Rajwanshi A, Srinivasan R, Nijhawan R. Fine needle aspiration of epididymal nodules in Chandigarh, north India: an audit of 228 cases. Cytopathology. 2006;17(4):195-198.

Correspondencia autor: Dr. J.E. Bestard Vallejo

Servicio de Urología. Hospital Universitario Vall d'Hebrón

Passeig de la Vall d'Hebrón, 119-129 - 08035 Barcelona

Tel.: 932746000

E-mail autor: 38625jbv@comb.es

Información artículo: Original - Andrología

Trabajo recibido: enero 2008

Trabajo aceptado: febrero 2008 